\title{
La primera indagación cubista de Picasso: Espacio interior I, II y III (Horta de Sant Joan y Barcelona, 1898-99)
}

\author{
Andrés Luque Teruel \\ Universidad de Sevilla
}

\begin{abstract}
RESUMEN: Una hoja con diversos dibujos de Picasso conservada en el Museo Picasso de Barcelona, titulada Niño y otros croquis, documentada en Horta de Sant Joan y Barcelona, en 1898-99, presenta tres interpretaciones sucesivas de un mismo espacio, variantes en las que se ensayaron y anticiparon la inversión espacial y la búsqueda de las nuevas fórmulas pictóricas que condujeron al cubismo. La revisión de ciertos aspectos de las pinturas, dibujos y apuntes de la época permite establecer conexiones que habían pasado inadvertidas y discutir con nuevas perspectivas la procedencia del cubismo.
\end{abstract}

PALABRAS CLAVE: Picasso, Origen de las vanguardias, Vanguardias, Cubismo.

Picasso's First Cubist Inquiry: Inner Space I, II and III (Horta de Sant Joan and Barcelona, 1898-99|

\begin{abstract}
A piece of paper with various Picasso drawings, kept at Picasso Museum in Barcelona and entitled Boy and other sketches, is based on Horta de San Juan and Barcelona in 1898-99. It presents three successive space interpretations which anticipated the search and spatial perception of Cubism. The review of certain aspects in the paintings, drawings and sketches of the period reveals connections that were otherwise unnoticed, and it approaches the origins of Cubism from new angles.
\end{abstract}

KEY WORDS: Picasso, Origins of the Vanguards, Avant-Garde Art, Cubism.

Recibido: 26 de febrero de 2014 / Aceptado: 30 de junio de 2014.

Cuando Picasso realizó y firmó la hoja de dibujo catalogada en el Museo Picasso de Barcelona con el título Niño y otros croquis ${ }^{1}$, en la que, entre estos, aparecen los pequeños apuntes que denominaremos aquí Espacio interior I, II y III, dejó un legado clave, hasta ahora inadvertido, para el análisis de la génesis cubista como un proceso complejo, debido a la esfera de los conceptos y al campo de esta relativo a los ensayos empíricos con los elementos formales preestablecidos, y no a la simpleza de una influencia exterior, la del "arte negro", que provocase una transformación inmediata, como es habitual en artistas menos dotados y sin su excepcional potencial creativo. Eso explica los continuos cambios formales,

* LUQUE TERUEL, Andrés: "La primera indagación cubista de Picasso: Espacio Interior I, II y III (Horta de Sant Joan y Barcelona, 1898-99)", Boletín de Arte, n. ${ }^{\circ} 35$, Departamento de Historia del Arte, Universidad de Málaga, 2014, pp. 187-205, ISSN: 0211-8483.

1 Museo Picasso, Barcelona (MPB). Pluma sepia y lápiz sobre papel, 32 × 24'5 cm. MPB 110577. 
supeditados a la realidad superior de ese sistema creativo que puede identificarse desde sus primeras obras y con independencia de los géneros y los estilos implícitos o derivados ${ }^{2}$. Lo que no quiere decir, como veremos, que tal estímulo no pudiese enriquecer el proceso, cuestión muy distinta al fundamento en sí.

\section{Síntesis de una cuestión abierta: el «arte negro» como origen o no del cubismo}

Antes del análisis de los croquis es preciso tener en cuenta la discusión historiográfica sobre el origen del cubismo, sistemáticamente obviada por los que se acercan al tema de modo ocasional y casi siempre con prejuicios y por la inmensa mayoría de los medios de difusión, que convirtieron una de las dos tendencias reconocidas, la procedencia del "arte negro», en una máxima categórica y, de ese modo, adulteraron una realidad historiográfica fundamental ${ }^{3}$. Lo hicieron con incomprensibles afanes positivistas que, en vez de establecer parámetros rigurosos, alejan del verdadero estado de la cuestión, amparados por la firma de algún autor anterior, casi siempre en su misma situación, obviando la obligación de valorar las distintas aportaciones, análisis e interpretaciones, y después pronunciarse al respecto con argumentos contundentes. Debe tenerse en cuenta el verdadero estado de la cuestión y no solo una parte del mismo, pues eso lleva a la deformación del problema y al establecimiento de pautas de conocimiento incorrectas por intencionadas.

Los primeros autores que escribieron sobre el cubismo no eran historiadores; mas sí escritores, críticos o artistas relacionados, fuentes directas, que

2 Entendido como un procedimiento de índole plástica acreditado en sus carnets con las múltiples variantes sobre una misma referencia formal, de las que obtenía nuevos puntos de partida para nuevas variaciones y obras posteriores y de distintas épocas. Esa forma de trabajar, basada en la interpretación intuitiva de unas cualidades originales, fuesen del natural, de la literatura o la imaginación, o de otras obras de arte, y las reflexiones implícitas, ya en los procesos de síntesis, ya en las elecciones para nuevas indagaciones formales, fue común en todas sus etapas, con independencia de las distintas circunstancias y de las motivaciones alternativas. De ahí la consideración de un sistema creativo personal. Véase: LUQUE TERUEL, Andrés, "Picasso, sistema creativo propio», Espacio y Tiempo, n. ${ }^{\circ} 21,2007$, pp. 109-135.

3 De este modo, los conceptos, rectores en una esfera intelectiva superior, se identifican o, al menos, relacionan, con el sistema creativo propio o procedimiento previo al definitivo de cada género, determinando un primer nivel creativo, del que procede el segundo, en el que significan los procedimientos de cada género y las formas, del que, a su vez, parte un tercer nivel, en cuyo ámbito adquieren identidad los estilos. La noción de estilo se explica en un tercer nivel creativo, siempre consecuente, es, pues, equivalente a un territorio, como tal, concreto, o la última consecuencia en el campo de las formas. Véase: LUQUE TERUEL, Andrés, "Consideración histórica del cubismo, origen del arte cubista en Las señoritas de Aviñón y nueva valoración de la pintura cubista de Picasso", Boletín del Museo e Instituto Camón Aznar, n. ${ }^{\circ}$ 99, 2007, pp. 309-366. 
vieron y compartieron tertulias y conversaciones con Picasso, Braque y los principales pintores y escultores adscritos al movimiento. Ninguno de ellos estableció vínculos determinantes con el "arte negro" y la mayoría no reparó siquiera en la existencia de este, cuyas obras, casi todas esculturas, se conservaban en el Museo del Trocadero, dedicado a las ciencias naturales y no al arte. Si alguno las citó fue como testimonio de algo raro, exótico, que despertó interés en una dimensión paralela al hecho creativo contemporáneo, sobre el que, por supuesto, tuvo una incidencia concreta una vez definidas las relaciones internas cubistas con conceptos complejos difíciles de discernir.

En ese grupo inicial hay que citar a Gelett Burgess ${ }^{4}$, Michel Puy ${ }^{5}$, Albert Gleizes y Jean Metzinguer ${ }^{6}$, André Salmon7, Guillaume Apollinaire ${ }^{8}$, Gertrude Stein, Paul Sérusier ${ }^{9}$, Daniel-Henri Kahnweiler ${ }^{10}$, Juan Gris ${ }^{11}$ y G. Janneau ${ }^{12}$, todos activos entre 1910 y 1930 . El rigor, la profundidad y el alcance fueron distintos en todos los casos. Casi todos situaron el origen del cubismo en las reflexiones plásticas y la creatividad técnica y formal de Picasso; alguno intentó apropiarse y adjudicarse sin éxito tal logro; y ninguno lo relacionó con el "arte negro". Incluso Alfred Barr, en un principio, optó por la consolidación, definitiva, de las categorías analítica y sintética y, como los anteriores, ni siquiera planteó tal posibilidad ${ }^{13}$; y poco después, Robert Goldwater lo vinculó con la evolución de la tendencia contemporánea primitiva, o arte primitivo contemporáneo ${ }^{14}$, lo que nos lleva a una ampliación del marco cronológico hasta 1938 sin que nadie propusiese ni insinuase el "arte negro" como origen ni elemento fundamental en la creación del cubismo.

4 BURGESS, Gelett, "The Wild Men of Paris", Architectural Record, Nueva York, 1910.

5 Cit. en GANTEFÜHER-TRIER, Anne, Cubismo, Colonia, Taschen, 2008, p. 8.

6 GLEIZES, Albert; METZINGER, Jean, Du Cubisme, París, 1912.

7 SALMON, André, Histoire anecdotique du cubisme, París, 1912. SALMON, André, La jeune peinture française, París, 1912.

8 APOLLINAIRE, Guillaume, Meditations esthétiques. Les peintres cubistas, París, Figuiére, 1913. APOLLINAIRE, Guillaume, "Picasso", Montjoie!, París, 14 de marzo de 1913.

9 DENIS, Maurice, ABC de la peinture. Étude sur la vie et L'CEuvre de Paul Sérusier, París, Floury, 1942, p. 100.

10 KAHNWEILER, Daniel-Henry, "Das Wesen der Bildhauerei», Feuer, año I, n. ${ }^{\circ} 2$ y 3, noviembre y diciembre de 1919, pp. 146 y ss. KAHNWEILER, Daniel-Henry, "Vom Sehen und vom Bilden», Weisse Bläter, n. ${ }^{\circ}$ 7, Berlín, julio de 1919. KAHNWEILER, Daniel-Henry, The rise of cubism, Nueva York, 1949.

11 GRIS, Juan, "Notas sobre mi pintura", Der Querschnitt, Fráncfort del Main, n. ${ }^{\circ} 1$ y 2, 1923, pp. 77 y 78. GRIS, Juan, Conferencia pronunciada ante el Grupo de Estudios Filosóficos y Científicos de la Sorbona, en el Anfiteatro Michelet, el día 15 de mayo de 1924, reproducida por KAHNWEILER, Daniel-Henry, Juan Gris. Sa vie, son oeuvre, ses écrits, París, Gallimard, 1946 (Barcelona, Quaderns Crema, 1995, pp. 434 y ss.).

12 JANNEAU, Guillaume, L'Art cubiste. Theories et realizations, Étude critique, París, 1929.

13 BARR, Alfred, Cubism and Abstract Art, Nueva York, MoMA 1936, pp. 15 y ss.

14 GOLDWATER, Robert, Primitivism in Modern Art, Nueva York, MoMA, 1938 y 1967. 
En ese contexto, Kahnweiler defendió durante décadas el origen del cubismo como consecuencia de las reflexiones plásticas de Picasso, y, una vez madurado este, la influencia del mismo y una nueva proyección teórica del entorno sobre la identificación o no del nuevo movimiento artístico con el sistema volumétrico de Cézanne y con el "arte negro». En ese debate, y no antes, situó el encuentro de Picasso y Braque y la evolución paralela que llevó a los períodos analítico y sintético ${ }^{15}$. La posibilidad de algún vínculo con Cézanne la redujo al período previo al cubismo, en 1906, y, respecto de la posible relación, estableció las enormes diferencias entre la geometría supeditada a la luz del francés y el nuevo espacio simbólico del español, cuyas relaciones formales en el plano estimó que rompen con la tradición y, por supuesto, con la reducción geométrica de Cézanne. Negó la influencia del "arte negro" en la creación del cubismo, y defendió justo lo contrario, que fue la creación original de Picasso la que posibilitó el ambiente necesario para la comprensión y la nueva valoración del arte étnico. Puso ejemplos del comercio de tales obras de arte como objetos exóticos a los que no se les daba valor artístico, incluida la publicitada máscara comprada por Vlaminck. Con las obras de Picasso, el mismo Kahnweiler y varios miembros de la vanguardia de Montmartre descubrieron los paralelismos de diversas esculturas exhibidas en el Museo del Trocadero. Fueron ellos los que reclamaron, por primera vez, la reconsideración del "arte negro" como tal ${ }^{16}$. No reconoció la influencia de este sobre Picasso hasta las esculturas del año 1913, nunca antes, pues según él, primero fue necesario el recorrido en el sentido indicado ${ }^{17}$.

Alfred Barr fue quien, avalado por la precisión histórica de sus estudios sobre el arte primitivo contemporáneo y el cubismo, propuso la nueva teoría de la influencia del "arte negro" en la creación del arte cubista, en Fyfty years of his $A r t^{18}$, en 1946. Esto sucedió pasados cuarenta años de la génesis del cubismo y sin que en todo ese tiempo ninguno de los protagonistas ni las fuentes directas o la crítica especializada hubiese dicho nada sobre ello. Alfred Barr dio una explicación genérica en un contexto general, y esta tuvo una amplia aceptación en los medios académicos franceses, complacidos con un hecho interesado, el movimiento adquiría así un nuevo origen francés a través de las colonias; aunque no aportó ningún documento ni análisis formal definitivo para establecer tales pautas, como una secuencia segura de variaciones a par-

15 KAHNWEILER, Juan Gris..., pp. 232 y 233.

16 Ibid., pp. 234-238.

17 Ibid., p. 233.

18 BARR, Alfred, Fyfty Years of his Art, Nueva York, MoMA, 1946, 1966 y 1974. 
tir de una referencia identificada, existente con otras fuentes, como la egipcia, la ibera y el natural. Su propuesta se quedó en la categoría de teoría, elevada a máxima por la repetición academicista que, desde entonces, ni analizó las fuentes en el orden cronológico correcto ni aportó análisis formales que lo corroborasen, limitándose a la identificación positivista de poses concretas con fotografías tribales, de las que Picasso tuvo una importante colección mas sólo pueden indicarse ligeras referencias compositivas y nunca aspectos conceptuales o formales determinantes.

Picasso, que siempre mantuvo oculta la naturaleza de su sistema creativo, nunca opinó al respecto, solo negó la influencia del "arte negro" y elogió públicamente la capacidad de Kahnweiler para comprender el Arte. Braque sí lo negó, con una declaración contundente en la que aseguró que Picasso y él experimentaron la búsqueda de un espacio nuevo, ajeno a cualquier estilo establecido, incluido el "arte negro" ${ }^{19}$. Tanto él como Kahnweiler encontraron importantes apoyos en las siguientes décadas en José Camón Aznar ${ }^{20}$, Pierre Daix ${ }^{21}$, Wilfried Wiegand ${ }^{22}$, Pierre Daix y Joan Rosselet ${ }^{23}$, Helene Seckel ${ }^{24}$ y Carsten-Peter Warnc$\mathrm{ke}^{25}$, con el que llegamos hasta nuestros días. Todos lo negaron y propusieron el origen en el ámbito de las codificaciones personales de Picasso, derivadas de la experimentación con elementos plásticos propios. Werner Spies ${ }^{26}$ lo tuvo tan claro como los anteriores, aunque intentase mantener cierto equilibrio y evitase pronunciarse de modo contundente al respecto.

En los últimos años y en otro contexto, Yve-Alain Bois ${ }^{27}$ y, por otra parte, Rosalind E. Krauss ${ }^{28}$ representaron una corriente historiográfica opuesta a la lectura evolutiva derivada de una influencia estilística concreta, fuese la negra o cualquier otra predeterminada, en la que la creatividad de Picasso es entendida como un paradigma lingüístico (plástico) que debe resolverse en la identidad

19 VALLIER, Dora, "Braque, la peinture et nous», Cahiers d'Art, XXIX, París, 1954, p. 15.

20 CAMÓN AZNAR, José, Picasso y el cubismo, Madrid, 1956.

21 DAIX, Pierre, "ll n'y a pas d'art négre dans Las demoiselles d'Avignon", Gazette des Beaux Arts, París, 1970, pp. 242-270. DAIX, Pierre, Le Journal de cubisme, Ginebra, 1982, pp. 43 y ss. y 98 y ss.

22 WIEGAND, Wilfried, Pablo Picasso, Reinbeck, 1973, p. 60.

23 DAIX, Pierre y ROSSELET, Joan, Le Cubisme de Picasso, Neuchatel, 1979, pp. 151 y ss.

24 SECKEL, Helene, Les Demoiselles d'Avignon, París, 1987 (Barcelona, Polígrafa, 1988, p. 56).

25 WARNCKE, Carsten-Peter, Picasso, Colonia,Taschen, 1992, vol. I, p. 153.

26 SPIES, Werner, Las esculturas de Picasso, Stuttgart (Barcelona, 1971). SPIES, Werner, La escultura de Picasso, Stuttgart, 1983 (Barcelona, 1989). SPIES, Werner, Picasso sculpteur, París, Editions du Centre Georges Pompidou, París, 2000.

27 BOIS, Yve-Alain, "Kahnweiler's Lesson», Representations, n. ${ }^{\circ}$ 18, primavera 1987, pp. 33-68. "The Semiology of Cubism", en RUBIN, William, Picasso and Braque. A Symposium, Nueva York, Museum of Modern Art, 1989 / Nueva York, Thames and Hudson, 1992, pp. 169-209.

28 KRAUSS, Rosalind E, "The Motivation of the Sign", en RUBIN, Picasso and Braque..., pp. 261-287; La originalidad de la vanguardia y otros mitos modernos, Madrid, Alianza Forma, 1996, pp. 39-59. 
de las relaciones internas de cada obra y las relaciones establecidas entre ellas. Con ese posicionamiento, matizaron o desecharon tópicos y convencionalismos con una gran capacidad reflexiva, estableciendo, como es lógico, un marco superior en el que puede replantearse la cuestión superando los determinismos. Ese orden intelectivo fue discutido por T. J. Clark ${ }^{29}$, opuesto a las formulaciones semiológicas, y capaz de proyectar de nuevo el debate en el campo de los estilos.

Por otra parte, John Golding ${ }^{30}$, moderado y tendente al equilibrio, $y$, de modo decidido, Herbert Read ${ }^{31}$ y Willian Rubin ${ }^{32}$, optaron por apoyar la teoría de Alfred Barr, también durante décadas y casi hasta la actualidad. De ese modo, la cuestión del cubismo como un movimiento artístico con sus variantes estilísticas, o, lo que no es lo mismo, interpretado como un simple estilo, se mantuvo abierta durante seis décadas.

\section{Una temprana revelación de principios en la serie de dibujos Espacio interior I, II, y III}

En ese contexto de reposición o, como mínimo, de reflexión y discusión sobre el verdadero origen del cubismo en los métodos o el sistema creativo propio y exclusivo de Picasso, adquiere un extraordinario valor la serie de variaciones sobre un mismo tema que aquí se presenta, primero porque siempre se han considerado simples croquis, meros apuntes sin importancia alguna, y nunca se han relacionado entre sí como una serie específica y, en consecuencia, no se han analizado ni interpretado en ese sentido, por lo que se ha obviado su verdadera naturaleza y, lo más importante, la evolución desde un modelo natural hasta una composición racional obtenida de la elaboración mental de dicho modelo, con lo que eso significa una vez asumido que el resultado final es una configuración codificada y analítica sin precedentes en la historia del arte que, con toda claridad, anticipa las pautas formales de lo que en poco en tiempo será el movimiento definitivo de las vanguardias artísticas internacionales, el arte cubista ${ }^{33}$.

29 CLARK, T. J., "Farewell to an idea", Episodes from a History of Modernism, New Haven, C.T. Yale University Press, 1999. Picasso and Truth from Cubism to Guernica, Princeton University Press, 2013. 30 GOLDING, John, "The Demoiselles d'Avignon», Burlington Magazine, vol. C, n. ${ }^{\circ} 662,1958$, pp. 155 y ss. GOLDING, John, Cubism. A History and an Analysis. 1907-14, Londres, 1959 y 1968, pp. 24 y ss. 31 READ, Herbert, A concise History of Modern Painting, Londres, Thames and Hudson, 1959, 1968 y 1988 (Barcelona, Ediciones del Serbal, 1984, pp. 67-87). READ, Herbert, Modern Sculpture, Londres, Thames and Hudson, 1964 (Barcelona, Destino, 1994, pp. 59 y ss.).

32 RUBIN, "La génesis de Les demoiselles d'Avignon"; en Les demoiselles d'Avignon..., pp. 35 y ss.

33 Entendido como un movimiento artístico sujeto a unos principios conceptuales capaces de determinar nuevos códigos formales, en los que el espacio y el volumen se funden en una misma dimensión, inédita en la historia del arte. Picasso fue el máximo responsable del primer nivel, intelectivo, antes 
1. Picasso, Niño y otros croquis, Horta de Sant Joan y Barcelona, 1898-99. Museo Picasso de Barcelona, MPB 110577

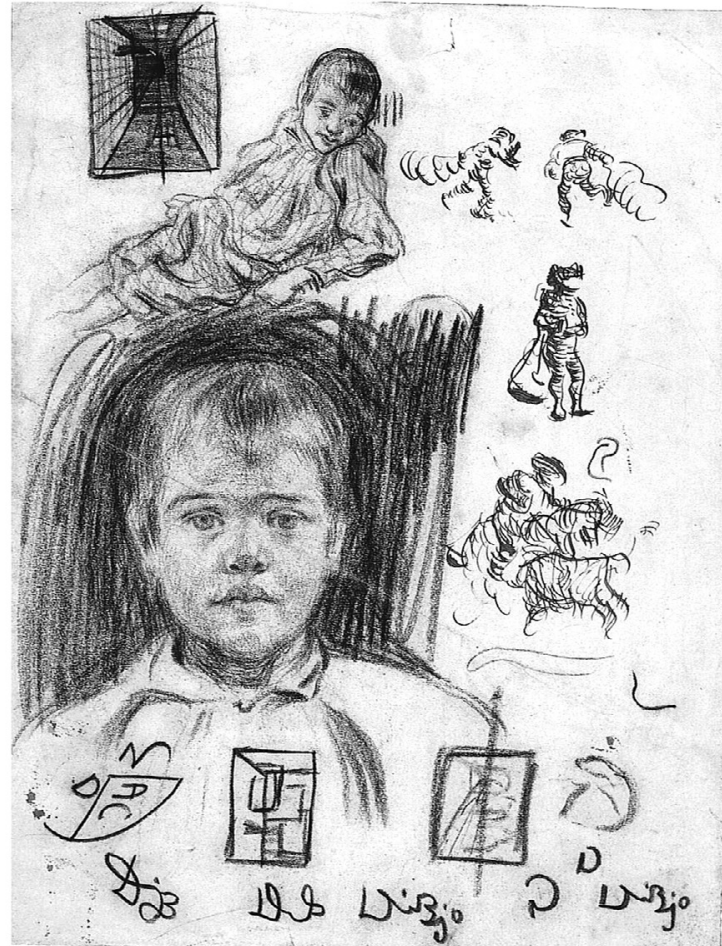

Los dibujos de Niño y otros croquis [1] ${ }^{34}$, realizados por Picasso en Horta de Sant Joan y Barcelona, en 1898-99, han tenido hasta ahora dispar consideración, pues el tema principal de la hoja, debido a su tamaño y definición, centró la atención de los expertos y los pequeños croquis que están a su alrededor quedaron relegados por un rango plástico menor, sin que nadie los estudiase de modo concreto, entre ellos los tres que forman la serie de variantes sobre un espacio interior aquí identificada como antecedente del cubismo. El pequeño tamaño, la simplificación formal y el desorden de la secuencia les restaba protagonismo en relación con la atractiva definición del busto infantil, y así pasaron desapercibidos o, al menos, nadie ofreció el análisis debido.

indicado; y él mismo secundado por Braque del segundo, en el ámbito de las formas. De esos códigos formales surgieron los estilos personales en un tercer nivel creativo, dependiente, desarrollados con mayor o menor fortuna por los distintos artistas vinculados.

34 En los márgenes superior e inferior aparecen una serie de croquis, tres de ellos relacionados entre sí, en los que se puede identificar una temprana anticipación de las relaciones internas características de las obras cubistas. 
Cuando Juan Manuel Bonet comentó que «sería sacar las cosas de quicio ver una prefiguración del cubismo" en los desnudos de esta época en los que Picasso asumió la correspondencia geométrica de la estructura del cuerpo, no se refería, como es evidente, a estos croquis en los que no aparece la figura huma$n a^{35}$. No hubo ningún rechazo por su parte a la identificación de esta pequeña y trascendental serie, simplemente, hablaba de otra cosa.

Claustre Rafart citó todos los dibujos de la hoja y se centró en tal figuración, que consideró una de las más brillantes síntesis del quehacer artístico de Picasso en esta época ${ }^{36}$. Incluso la publicó, por lo que está al alcance de todos, sin que nadie hasta ahora haya reparado en la importancia de los pequeños croquis en los que Picasso anticipó los conceptos y criterios plásticos cubistas, por lo que, en ese sentido, deben considerarse inéditos. Tampoco fue su propósito el análisis de estos, sino el de la estructura del cuerpo en el dibujo figurativo principal.

Picasso dibujó en la parte superior de la hoja una habitación en perspectiva, ligeramente desplazada hacia la derecha del formato. Podríamos denominarla Espacio interior I [2] ${ }^{37}$. Su reducido tamaño y la precisión y la simpleza con la que representó las líneas fundamentales para la fuga y la definición de la ventana que la desplaza desde un punto de vista sensorial hacen que, a simple vista, carezca de importancia. En la parte inferior de la hoja hay otras dos pequeñas composiciones acotadas en un formato rectangular, Espacio interior II y Espacio interior III, en las que experimentó con una simplificación extrema de la vista en perspectiva de la parte superior, organizada en planos mediante cruces de líneas verticales y horizontales, como las de los armazones del cubismo analítico más avanzado. Al lado de estas, en el margen izquierdo del formato, una pequeña composición curvilínea no se muestra como un antecedente tan directo y fácil de identificar en su proceso evolutivo; mas recuerda las relaciones de curvas y contra curvas de esculturas como Mujer sentada, en 1907, y los espacios llenos y vacíos de las esculturas cubistas avanzadas.

La secuencia establecida por los tres croquis que forman la serie de variantes sobre un mismo tema, sistema creativo habitual de Picasso en su amplia carrera,

35 BONET, Juan Manuel, "Barcelona, fábrica de dibujos, o el dibujo como laboratorio" en Picasso, la formación de un genio, 1890-1904, Barcelona, Museu Picasso, 1997, p. 20.

36 RAFART, Claustre, "El rechazo a la Academia y el anhelo de modernidad: Madrid 1897, Barcelona 1899 ", en AA. VV., Picasso. La formación de un genio..., p. 120.

37 Secuencia ordenada de arriba abajo y de izquierda a derecha de los croquis que aparecen en los márgenes de Niño y otros croquis. La primera variante reproduce el espacio en perspectiva con criterios naturalistas; la segunda tiende a la simplificación radical y la codificación de los elementos plásticos resultantes; la tercera presenta una configuración codificada, en la que el espacio y las formas se proyectan en un mismo y novedoso plano y en función de distintos rayos centrales de visión, argumentos esenciales del futuro arte cubista. 

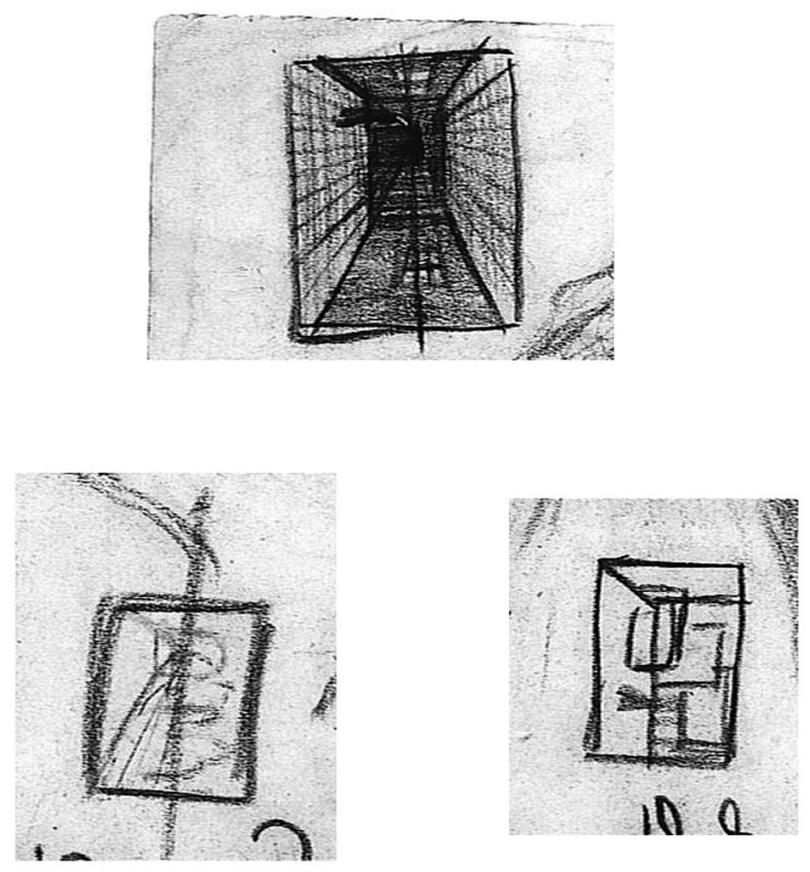

2. Picasso, Espacio Interior I, II y III

debe interpretarse de arriba abajo y en el sentido de las agujas del reloj. El primer dibujo es un apunte naturalista, con un estudio de perspectiva bien resuelto, nítido, claro y sin ningún tipo de error. El segundo mantiene referencias suficientes para identificarlo con el anterior. La diferencia está en la mayor simplificación y cierta tendencia, no resuelta, a la eliminación del espacio simbólico tradicional y su sustitución por nuevas reglas plásticas que remiten a dicha realidad sin representarla de un modo físico, sino con claves racionales supeditadas a las nuevas relaciones artísticas. El tercero de los dibujos es definitivo, la estructura, casi abstracta, permite identificar líneas análogas a las del segundo, hecho que muestra la continuidad, y, siguiendo la secuencia a la inversa, del primero. En este, el espacio y los volúmenes desplazados de la anterior fuga, ocupan un mismo y único plano, en una dimensión superficial inédita. Las líneas y los planos determinados forman una estructura con verticales y horizontales que generan campos asociados, a los que sólo faltan fondos cromáticos o contrastes de luces y sombras para que sea igual que cualquier pintura o dibujo cubista analítico. 
El análisis formal no deja dudas, nadie podrá negar el carácter sucesivo de los croquis, ni que las variaciones sobre un mismo tema pasan gradualmente de la interpretación del natural a los esquemas mentales, racionales y casi abstractos, en los que Picasso definió un nuevo espacio pictórico, plástico y de índole intelectiva y sin vínculos visuales directos con la realidad. Todo esto origina una serie de interrogantes, como ¿fue Picasso consciente en ese momento de la trascendencia de su nueva configuración o del potencial que tenía? ¿Tuvo alguna incidencia inmediata en otras alternativas creativas del propio artista de la misma época? ¿Por qué relegó durante años su gran hallazgo? Y, aunque la respuesta de esta es sabida, es lógico replantearla aquí, ¿lo hizo en otras ocasiones y épocas?

La respuesta a la primera pregunta es subjetiva, nadie puede asegurar si Picasso fue consciente o no en aquel momento de la importancia del nuevo arte reflexivo y racional que proponía, solo podemos pensarlo, deducirlo o creerlo; sin embargo, sí es seguro que siempre tuvo muy claras dos cuestiones: la importancia de las series de variantes como procedimiento reflexivo en el que la experimentación e incluso el azar juegan un papel fundamental; y la vigencia intemporal de cualquier hallazgo formal, por sorprendente, inaudito o baldío que pudiese parecer en su momento. Para Picasso no había callejones sin salida, todo lo que fuese una indagación propia algún día podría servir para algo, tener algún sentido, ya como recurso o parte de una composición compleja en la que estuviesen implicados los resultados de otras aplicaciones del sistema, esto es, de otras indagaciones formales con sus respectivas decisiones y relaciones; ya como fundamento en sí de la nueva obra.

Así lo reconocieron numerosos autores, como Kahnweiler, Wilfried Wiegand, Werner Spies, Pierre Daix, Carsten-Peter Warncke y, como ejemplo reciente, Claustre Rafart, que ya dedujo que, desde época juvenil, «de la maduración de uno o varios dibujos surgía a veces una pintura». ${ }^{38}$ Así que, fuese consciente o no de la importancia de la serie, es seguro que estaba convencido de que algún día pudiera servirle para algo, aunque en ese momento no supiese para qué ni cómo.

La segunda pregunta, sobre la incidencia o influencia en otras obras de la época, está condicionada por el reconocimiento del sistema creativo de Picasso, por la seguridad del origen del proceso intelectivo desde una referencia natural y no del "arte negro" y por la relación admitida en la anterior. Solo así se podrá interpretar con un nuevo y adecuado sentido el alcance de los ejercicios geomé-

38 RAFART, Claustre, "El rechazo a la Academia y el anhelo de modernidad: Madrid 1897, Barcelona 1899", en AA. VV., Picasso. La formación de un genio..., p. 119. 
tricos proyectados en elementos o zonas concretas de numerosas figuraciones de la época. Eso no quiere decir que tales ejercicios de depuración insinúen siquiera las pautas cubistas, como afirmó con acierto Juan Manuel Bonet; mas sí que forman parte de un mismo contexto que las variantes de Espacio interior I a III, con las que, al menos, comparten los intereses racionales que Ilevan a cierta autonomía esencialista en las relaciones que mantienen en sus respectivas configuraciones, estas regidas por criterios visuales en los que se integran como un elemento más.

Según lo expuesto, y en relación con la tercera pregunta, no es que relegase durante años el hallazgo, sino que, como demostraron numerosos autores en otros casos, su caudal reflexivo y experimental era tan prolífico que produjo una excepcional acumulación de posibilidades creativas y plásticas en la esfera de las formas, superior a la de los estilos, secundarios para él. Esa acumulación le sirvió para recuperar el planteamiento o la forma que considerase oportuna en el momento adecuado para ello. Por lo tanto, no hay un salto cronológico, sino, como dijo Malén Gual sobre la proyección de los dibujos de Barcelona respecto de la evolución propia en París, Picasso «capta detalles con espontaneidad proyectándolos en realizaciones posteriores" ${ }^{39}$.

Ahora, la cuarta pregunta planteada, cuya respuesta es de sobra conocida, adquiere un nuevo sentido: Picasso recurrió a conceptos, formas y variantes formales de otras épocas, incluso muy lejanas en el tiempo, durante toda su carrera. Fue una constante en la aplicación del sistema propio muy definido que, con esta serie de croquis, queda, una vez más, suficientemente claro, pues demuestra el sentido de la experimentación formal y la reflexión conceptual pareja y consecuente y el valor de la serie y sus variantes en el proceso creativo que lleva a una obra definitiva. Cada desarrollo partió de una referencia, como ya se ha demostrado con originales egipcios e iberos respecto de la configuración de algunas figuras de grandes composiciones cubistas, como Las señoritas de Aviñón, y esta referencia determinó, con sus cualidades innatas, la categoría formal específica de partida y el sentido de las variaciones que llevaron a resultados sorprendentes y dispares.

Esa aplicación es la que condujo a Picasso a la definición del arte cubista, y no una influencia externa ni la ascendencia de un estilo determinado, por supuesto en un camino complejo, con múltiples referencias sobre las que aplicar tales propósitos en la búsqueda del nuevo espacio pictórico después de las suce-

39 GUAL, Malén, «El primer contacto con la vanguardia. Barcelona 1899-1900», Ibid., pp. 185 y 186. 
sivas depuraciones formales de los períodos azul y rosa, de 1902 a 1905, y, sobre todo, primitivo, en 1906. En ese momento, cuando buscó, por otros motivos y en el contexto de las vanguardias de Montmartre, la pureza del arte de los primeros tiempos, con independencia de la carga de significado original y siempre en función de medios expresivos personales, de los que derivarán los estilos, estuvo preparado para volver la vista atrás y recuperar el proceso analítico que ahora se demuestra efectuó por primera vez en Horta de San Juan y Barcelona, en 1898-99, mucho antes de su primer viaje a París y, por lo tanto, de que tuviese conocimiento de la existencia del "arte negro".

Numerosas fotografías tribales conservadas por Picasso lo mantienen como referencia sobre la que trabajó en numerosas ocasiones en época posterior a Espacio interior I, II, y III, con independencia del momento creativo del artista, debido siempre a la aplicación de su sistema. Este condicionó la influencia que los modelos negros pudieron ejercer en las sucesivas etapas de Picasso, con sus respectivas definiciones formales y estilos. Se pueden poner ejemplos significativos, como la pintura y el gouache de la etapa rosa titulados Los dos hermanos ${ }^{40}$, en Gósol, en 1906, en las que uno aparece como porteador al modo africano, circunstancia desapercibida por el inmenso clasicismo formal y la moderna y bella depuración de los desnudos; y Mujer de perfil ${ }^{41}$, en 1906-07, pintura primitiva y cercana al cubismo, en la que repitió la postura de una de las fotografías de su colección.

\section{Un nuevo enfoque: antecedentes que revirtieron en el proceso que generó el cubismo}

El reconocimiento de Espacio interior I a III como una experimentación precubista remite el origen del movimiento a unas coordenadas distintas y anteriores a la de su definición en la vanguardia de París. Esas coordenadas y otras inquietudes confluyentes, todavía alternativas en 1898, pueden apreciarse en numerosos dibujos y algunas pinturas realizadas por Picasso en Barcelona y otras localidades catalanas; e igualmente se identifican en Manos del artista dibujando a Francisco Bernareggi en un papel frente a é ${ }^{42}$, dibujo realizado en Madrid el día 17 de mayo

40 Museo de Bellas Artes de Basilea, óleo sobre lienzo, 142 x 97 cm. Z 1, 304; DB XV, 9; P 1233. Musée Picasso, París, gouache sobre cartón, 80 x 59 cm. Z 6, 720; DB XV, 8; P 1229.

41 Colección particular. Óleo sobre lienzo, 75 x $53 \mathrm{~cm}$. GANTEFÜHRER-TRIER, Cubismo..., p. 8.

42 Tinta y lápiz sobre papel. MPB 111340. 
3. Picasso, Retrato de José Ruiz Blasco, Barcelona, 1898-99

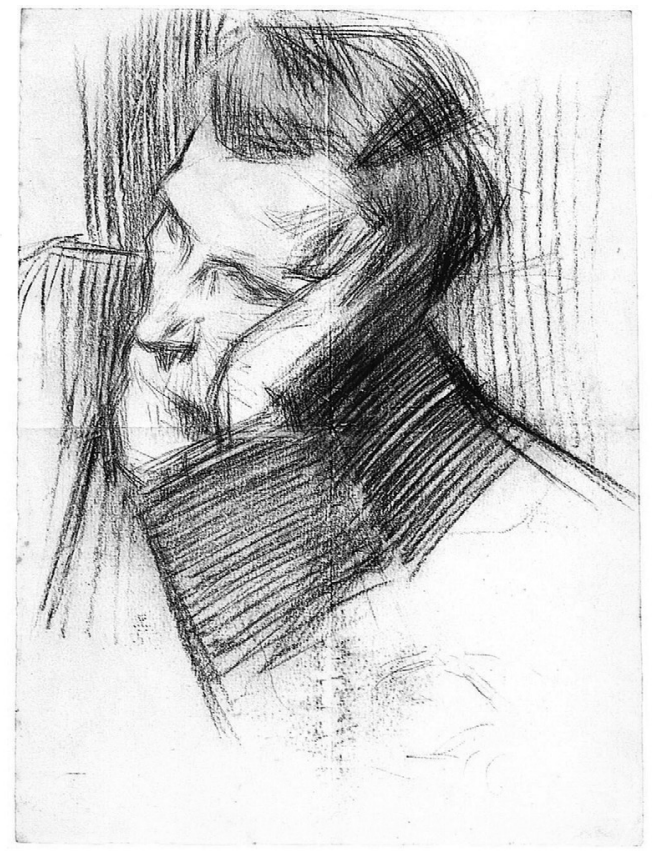

de1898. Teniendo en cuenta la identificación aquí propuesta, puede decirse que las concibió como antecedentes categóricos de una evolución inminente y radical, hasta el punto que María Teresa Ocaña afirmó lo siguiente: «Ningún dibujo del año 1901 pretende transmitir una idea placentera de la realidad, subyace un componente crítico» ${ }^{43}$.

Rosa Vives advirtió, con sumo acierto, la novedad de Picasso en la utilización del plumeado, planteado como técnica en la que las masas compactas de líneas paralelas, cuyo objetivo original es sombrear una superficie, proporcionan un concepto geométrico del dibujo en relación con un plano determinado. Por derivación, pensó que, "mantenido en un conjunto geometrizante», la relación de planos plumeados configura una estructura analítica que indica de manera sintética y vigorosa las zonas de luz y sombra, sin medias tintas. Puso como ejemplo el Retrato de José Ruiz Blasco [3] ${ }^{44}$, dibujado en Barcelona en 1898-99,

43 OCAÑA, María Teresa, "Los años de la eclosión: Barcelona-Madrid-París, 1900-1904», en AA. VV., Picasso. La formación de un genio..., p. 243.

44 Lápiz sobre papel, $22 \times 16^{\prime} 4 \mathrm{~cm}$. MPB 110726. Recursos tradicionales como el plumeado adquirieron un nuevo tratamiento en planos superpuestos, afín a las integraciones analíticas y sintéticas desarrolladas en Espacio interior II y III. 
que consideró impactante por la amplitud y la concisión de los bloques estructurales que marcan los planos de luz y sombra ${ }^{45}$.

Aunque no los nombrase, la deducción de Rosa Vives relaciona la intención de los planos plumeados de Picasso en la configuración de determinadas obras naturalistas con la racionalización de Espacio interior II y III, y con argumentos definitivos de la etapa primitiva más acusada del artista y el origen del cubismo.

Lluís Bagunyá aseguró en consonancia con todo ello ${ }^{46}$ :

En esos años, aparte de su dibujo académico, Picasso experimentó técnicas y trazos, líneas rectas, haces curvas, bocetos realizados sin levantar la pluma... prácticamente todo es susceptible de ser asumido como una técnica propia.

Algunos ejemplos de esa creatividad técnica fundamental pasaron desapercibidos como partes concretas de obras que deslumbraron por otras cualidades, como sucede en Dos figuras femeninas [4] ${ }^{47}$, en Barcelona, en 1899-1900; y Mujer desnuda sentada [5] $]^{48}$, en Barcelona, en 1899, bella obra de transición hacia las nuevas preocupaciones vanguardistas. En esta acaparó la atención el naturalismo, en principio con claras concesiones academicistas en la pose de la modelo; en segunda instancia, muy moderno por la esquematización y la organización estructural de los trazos. Si lo analizamos con profundidad, un elemento clave reclama la atención, la sombra del pecho izquierdo, codificada con un trazo en ángulo, está resuelta con doble intención, marca un plano oscurecido más que define un volumen, y se muestra como un signo plástico en sí misma, con la contundencia de las soluciones primitivas que algunos identificaron de modo incorrecto con el "arte negro". El gato sentado debajo del banco alto muestra codificaciones análogas. El interés por la sombra como

45 VIVES, Rosa, "Desafío en la hoja de papel», en ,Picasso. La formación de un genio..., p. 36.

46 BAGUNYÁ, Lluís, "La formación académica y el aprendizaje: Málaga 1890-Barcelona 1897», en ibid., p. 54.

47 Lápiz sobre papel, $14^{\prime} 7 \times 16^{\prime} 5 \mathrm{~cm}$. MPB 110840R. Una vez identificados los fundamentos que generaron el cubismo en la serie de croquis Espacio interior I, II y III, puede asegurarse que este procede de la experimentación y la elevación conceptual de Picasso, no de la referencia directa del "arte negro». Siendo así, es preciso valorar de nuevo la creatividad técnica de Picasso, pues muchas decisiones tempranas reaparecieron en unos años con las lógicas transformaciones evolutivas como soluciones complementarias en sus propuestas primitivas y en el inicio del cubismo como movimiento estético en París. El sombreado de la nariz de este busto procede de esa racionalización, verdadero origen de los rasgos que después fueron confundidos como aportaciones del "arte negro".

48 Lápiz sobre papel, 47'6 × 31'6 cm. MPB 110594. La sombra angular de debajo del pecho debe considerarse el auténtico punto de partida para los trazos agresivos de los personajes femeninos de las señoritas de Aviñón, y no el "arte negro". 


\section{1: artículos La primera indagación cubista de Picasso...}

4. Picasso, Figuras

femeninas, Barcelona,

1899-1900

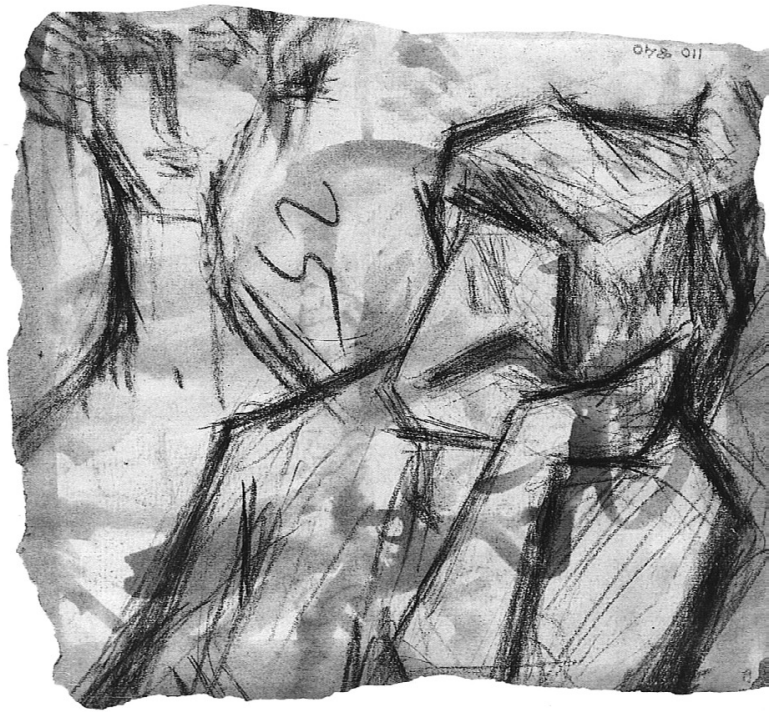

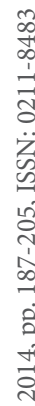

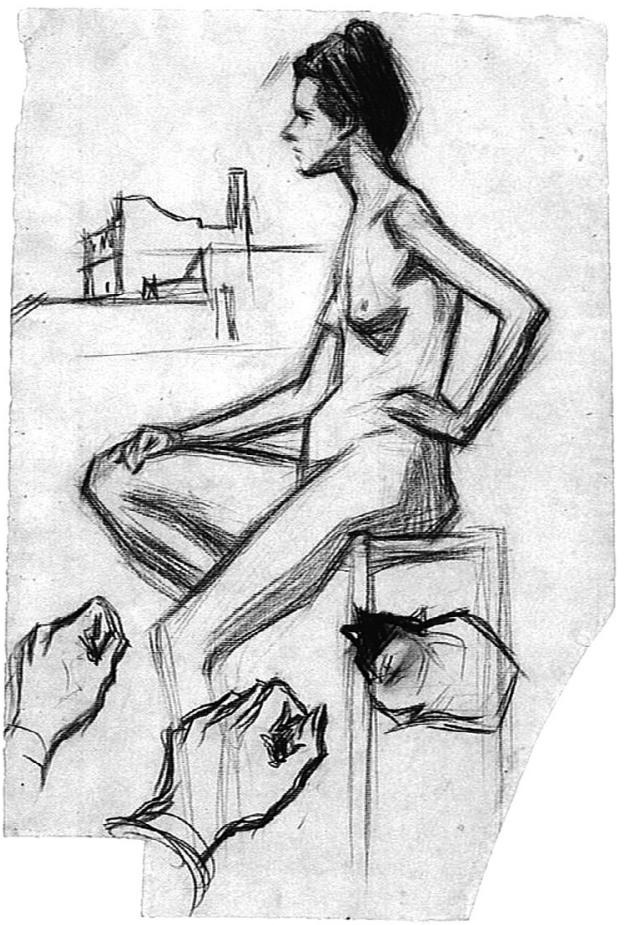

m

I

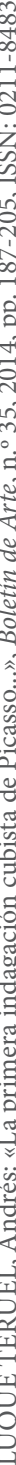

desnuda sentada, Barcelona, 1899 
rayado que la supone está también insinuado en Desnudo femenino de perfi/4, en Barcelona, en 1899, en este caso en la zona inguinal y bajo el pecho izquierdo, aunque de un modo bastante más moderado pese a al grosor y la fuerza de los trazos.

Esos recursos pasaron de estas obras a algunas pinturas rosas, a bastantes de las primitivas y, de estas, a las primeras cubistas. De ahí su importancia, nunca reclamada con la contundencia necesaria. Pueden verse múltiples casos, como en el Retrato de Carlos Casagemas [6 ${ }^{50}$, en Barcelona, en 1899-1900. Los trazos en ángulo, marcando las sombras bajo los ojos y en los laterales de la nariz son análogos a los que algunos dedujeron del "arte negro». El dibujo está roto y le falta la parte superior izquierda de la cara, aún así se aprecia el inicio de la sombra bajo ese ojo y en la otra y la nariz de modo completo. Los planos superpuestos concuerdan con las simplificaciones esquemáticas de la boca, y pudiera decirse que obedecen a una simplificación radical de la figura; mas la colocación de las sombras y el modo de yuxtaponerlas son idénticos a los de las pinturas primitivas y la serie de Las señoritas de Aviñón con la que Picasso inició el cubismo. Las sombras y los volúmenes se proyectan a la vez en el plano, sobre todo en la alternancia de la nariz. Ese mismo recurso aparece con criterios pictóricos y al servicio de la interpretación natural en Retrato de Carles Casagemas ${ }^{51}$ y Autorretrato ${ }^{52}$, los dos en Barcelona, en 1899-1900.

\section{Conclusión: conceptos y elementos cubistas de Picasso antes del cubismo}

La conclusión de mayor alcance es la certeza de la naturaleza conceptual y plástica de dos croquis de la serie, Espacio interior II y III, definidos por la configuración del nuevo espacio codificado en el plano que, con los matices y las salvedades expuestas, pueden considerarse ensayos embrionarios del Arte Cubista, debidos solo y exclusivamente a la creatividad técnica y formal de Picasso, años antes de que viajase a París y tuviese noticia de la existencia del "arte negro". Este tuvo su importancia como una referencia o aportación más, junto al natural, el arte prehistórico europeo, el egipcio, el ibero y el románico, en la búsqueda de un arte primitivo entre 1904 y 1906 y, llegado el momento, en la evolución del

49 Lápiz sobre papel, $31^{\prime} 5 \times 21^{\prime} 8 \mathrm{~cm}$. MPB 110340.

50 Lápiz sobre papel rayado, 30’5 x 19'9 cm. MPB 110653.

51 Óleo sobre lienzo, 55 x $45 \mathrm{~cm}$. MPB 110022.

52 Tinta sepia y acuarela sobre papel, $20^{\prime} 7 \times 13^{\prime} 1 \mathrm{~cm}$. MPB 110300. 
6. Picasso, Retrato de Carlos Casagemas, Barcelona, 1899-1900

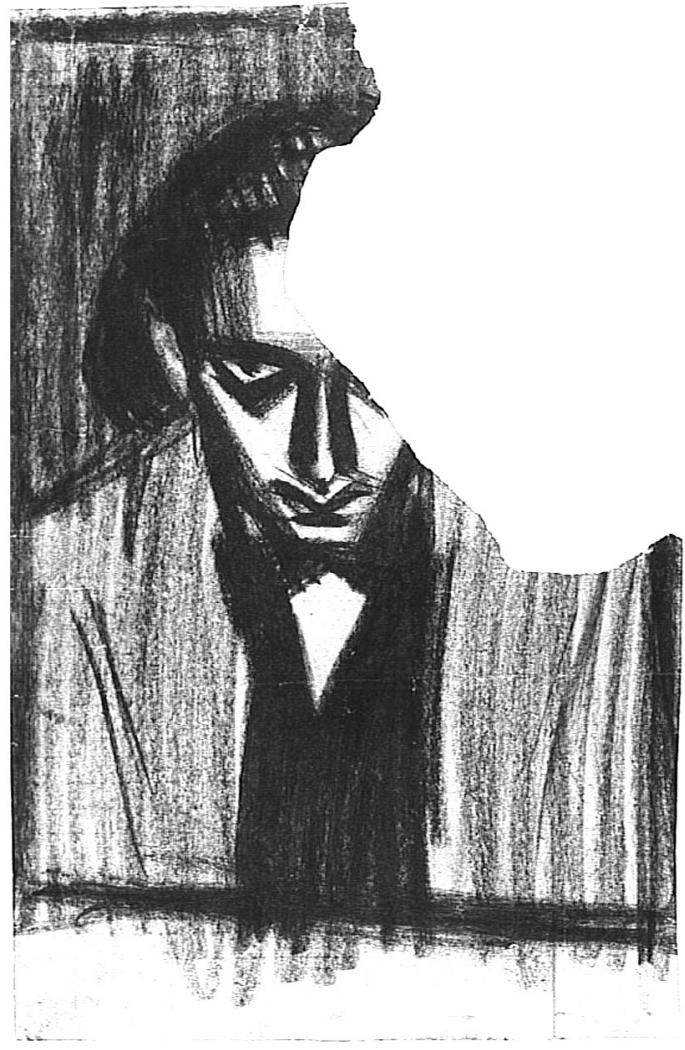


Eso lo llevó a un realismo extremo en París, con obras como Cabeza de muchacho ${ }^{53}$ y Cabeza mirando hacia lo alto ${ }^{54}$, las dos en 1902, con las que llegó a la pintura azul incorporando una nueva e intensa carga simbólica y social. La transición a la pintura rosa es bien conocida, como la de esta a la de la etapa primitiva derivada, momento este en el que, con la confluencia de conceptos, pudo recuperar las codificaciones fundamentales propuestas en Espacio interior I/ y III, y cuantos recursos de aquella época consideró necesarios por su novedosa y radical creatividad técnica.

Uno claro fue el de la independencia de distintos rayos centrales de visión, derivado del arte ibero, principio, como se dijo, fundamental en el arte cubista. La gran diferencia está en que en un primer momento lo vimos en la configuración de una misma imagen o espacio, y desde finales de la etapa rosa lo utilizó en la representación de dos imágenes distintas y yuxtapuestas de un mismo modelo, como aparecen en los dibujos Dos mujeres desnudas ${ }^{55}$; Dos mujeres desnudas ${ }^{56}$; y Mujer desnuda sentada y mujer desnuda en pies ${ }^{57}$, los tres en París, en 1906; y las pinturas Mujer desnuda de frente y desnudo de perfip8; y Dos mujeres desnudas ${ }^{59}$, las dos en París, a finales de 1906.

Recursos tradicionales, como el plumeado, adquirieron con Picasso un nuevo tratamiento en planos superpuestos, afín a las integraciones analíticas y sintéticas desarrolladas en Espacio interior II y III. Eso lo llevó a las estructuras cubistas facetadas que, según esto, por sí mismas, como tales soportes internos, no tienen justificación ni en el "arte negro" ni en ninguna tendencia primitiva o de cualquier época anterior. Los plumeados y las sombras derivadas o relacionadas y alteradas desde un punto de vista técnico asociadas a la nariz, que vimos en un número significativo de dibujos, fue determinante también en el cubismo. Desde el principio, pues fue uno de los criterios habituales y determinantes en la serie preparatoria de Las señoritas de Aviñón, como se aprecia en Mujer ${ }^{60}$, en París, en 1907. Los trazos firmes de la nariz propician tres espacios o planos. La estructura del cuerpo mediante diagonales y cruces de trazos, sobre el fondo

53 Lápiz sobre papel, $31^{\prime} 2 \times 13^{\prime} 5 \mathrm{~cm}$. MPB 110529.

54 Lápiz sobre papel, 31'2 2 24'3 cm. MPB 110530.

55 Lápiz sobre papel, 63 x $47 \mathrm{~cm}$. Zervos (Z) I, 365; Palau (P) 1388.

56 Colección Heinz Berggruen, Ginebra. Z I, 359; P 1390; DAIX, Pierre; BOUDAIILE, G (DB), Picasso: The Blue and Rose Periods. A Catalogue Raisonné of the Paintings, Londres, 1967. DB XVI, 21.

57 Museo de Bellas Artes de Philadephia, Colección Arensberg. Lápiz sobre papel, $63^{\prime} 6 \times 47^{\prime} 6 \mathrm{~cm}$. Z I, 368; P 1406; DB XVI, 17.

58 Colección Walter D. Floersheimer, Locarno. Aguada sobre papel, sobre lienzo, $58^{\prime} 5 \times 53^{\prime} 2 \mathrm{~cm} . \mathrm{Z}$ I, 336; P 1363; DB XVI, 7.

59 MoMA, Nueva York. Óleo sobre lienzo, 151'3 x $93 \mathrm{~cm}$. ZI, 366; P 1411; DB XVI, 15.

60 Colección Beyeler, Basilea. Óleo sobre lienzo, 119 x 93 cm. Z II, 631; P 1552; DR 75. 
blanco; los colores intensos de la parte superior, sobre todo el verde sobre el que emerge la silueta y el amarillo intenso del cuerpo y el fondo, centran la atención y la desvían de esa constitución constructiva que ya se vio en los dibujos de Barcelona y Madrid.

Esos sombreados, tenidos como la primera referencia detectable procedente del "arte negro" en Picasso, como los anteriores recursos técnicos o soluciones plásticas, fueron utilizados por este en cualquiera de los planteamientos formales y estilos entre los que fluctuó en pocos años, por lo tanto, no pudo ser nunca una simple cuestión estilística. En dos dibujos titulados Cabeza de mujer ${ }^{61}$, en París, en el invierno de 1906-07, están aplicados con criterios naturalistas con la misma efectividad que en cualquiera de las codificaciones previas al cubismo o en las obras cubistas más avanzadas. La misma solución, con trazos codificados y presencia plástica autónoma aparece en los siguientes dibujos: Cabeza de mujer62, en junio-julio de 1906; y Estudio para Las señoritas de Aviñón ${ }^{63}$, en marzo de 1907; y en las pinturas: Mujer desnuda ${ }^{64}$, Busto de mujer ${ }^{65}$, Cabeza $^{66}$, Busto de mujer $^{67}$ y Busto de mujer ${ }^{68}$, las cinco en París, en la primavera-verano de 1907; La danza de los velos, desnudo con paños ${ }^{69}$, y Desnudo, La bailarina de Aviñón ${ }^{70}$, en el verano-otoño de 1907; y La ofrenda ${ }^{71}$, en París, en 1908.

En bastantes aspectos, los trazos sueltos de Estudio para Las señoritas de Aviñón ${ }^{72}$, en París, en junio-julio de 1907, con verticales, horizontales y diagonales autónomas, desvinculadas de la figura humana, están más cercanos a los dibujos de Barcelona y Madrid que de la estructura cubista que anticipan. La relación no es circunstancial ni casual, todo lo contrario, es tan directa como en la configuración del nuevo espacio codificado en el plano de Espacio interior II y III, auténticos ensayos embrionarios del arte cubista.

61 Herederos del artista. Lápiz sobre papel, 32 × 24 cm. Z VI, 855; P 1481. Museo Picasso de París (MPP). Lápiz sobre papel, $14^{\prime} 7$ x 10'6 cm. Z II, 603; P1479; MPP 1859.

62 Colección Particular. Z VI, 968; P 1484.

63 Museo de Bellas Artes, Basilea. Z II, 19; P 1554; MPP 1861.

64 Colección Heinz Berggruen, Ginebra. Óleo sobre lienzo, 81 x 60 cm. Z II, 24; P 1550; DR 41.

65 Centro Georges Pompidou, París. Óleo sobre lienzo, 65 x 58 cm. Z II, 23; P 1556; DR 38.

66 Museo de Bellas Artes, Basilea. Óleo sobre lienzo, 96 × $33 \mathrm{~cm}$. Z II, 22; P 1555; DR 46.

67 Museo Picasso, París. Óleo sobre cartón, 53'5 x 36'2 cm. DR 28; MPP 15.

68 Galería Národni, Praga. Óleo sobre lienzo, 64’5 x 50 cm. Z II, 16; P 1546; DR 33.

69 Museo del Ermitage, San Petersburgo. Óleo sobre lienzo, $152 \times 101 \mathrm{~cm}$. Z II, 47; DR 95.

70 Colección Particular. Óleo sobre lienzo, 150'3 x 100'3 cm. Z II, 35; DR 53.

71 Gouache sobe papel, 30'8 x 31'1 cm París. MPB 112761.

72 Museo de Bellas Artes de Philadelphia. Lápiz sobre papel, 31'2 x 24'7 cm. Z VI, 936; P 1466. 
